\title{
High frequency distribution of heterogeneous vancomycin resistant Enterococcous faecium (VREfm) in Iranian hospitals
}

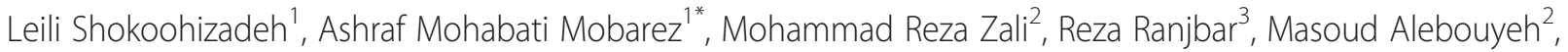 \\ Türkan Sakinc ${ }^{4}$ and Liaqat $\mathrm{Ali}^{4,5}$
}

\begin{abstract}
Background: Enterococcus faecium is a multi-resistant nosocomial pathogen causing infection in debilitated patients. Vancomycin-resistant enterococcus faecium (VREfm) are a major concern and increased dramatically worldwide especially in hospitals environment. The current study focused on determining the high prevalence and distribution patterns of antibiotic resistance and also its genetic linkages among various VREfm strains isolated from indoor hospitalized patients in four major Iranian teaching hospitals of Tehran.
\end{abstract}

Methods: The clinical samples were obtained from hospitalized patients during September 2010 to June 2011 from different teaching hospitals of Tehran. Antibiotics Resistance patterns, minimum inhibition concentration (MIC) value for vancomycin, ampicillin, gentamicin and presence of genetic linkage among the isolates were determined by pulsed-field gel electrophoresis (PFGE).

Results: Overall, total of $92(41.4 \%)$ isolates were identified as E. faecium, 45 (49\%) were resistant to vancomycin with an $\mathrm{MIC}_{50}$ of $\geq 128 \mathrm{mg} / \mathrm{L}$. The results showed that simultaneous resistance to teicoplanin, ampicillin, gentamicin, ciprofloxacine, tetracycline and erythromycin were observed the most frequent pattern. All the vancomycin resistant E. faecium isolates carried the vanA gene. intensive care units (ICUs) and Kidney transplantation, are most probably the wards with highest risk of infection by VRE. 17 pulsotypes were also detected by PFGE, most of the related pulsotypes belongs to the same hospitals.

Conclusions: This study shows the high alarming prevalence of Enterococcus faecium infection and similar clones of VREfm strains in Iranian hospitals with threatening resistance phenotypes.

Virtual slides: The virtual slides for this article can be found here: http://www.diagnosticpathology.diagnomx.eu/vs/ 1270863903102282

Keywords: E. faecium, Vancomycin resistant, Hospital and PFGE

\section{Background}

Enterococci have been regarded as low grade pathogens in the past, but in recent years it has rehabilitated into major pathogen in nosocomial infections [1]. Urinary tract and surgical site infections, bacteremia and endocarditis in immunocomprised patients are frequently caused by these bacteria in hospital [2]. The best therapy for enterococcal infection is the combination of aminoglycoside

\footnotetext{
*Correspondence: mmmobarez@modares.ac.ir

'Department of Bacteriology, Faculty of Medical Sciences, Tarbiat Modares University, P. O. Box: 14115-111, Tehran, Iran

Full list of author information is available at the end of the article
}

antibiotic (e.g. gentamicin) with a $\beta$-lactam antibiotic (e.g. ampicillin) or glycopeptides (e.g. vancomycin). However, vancomycin resistant enterococci (VRE) do not respond to these medications and thus there is limited option of treatment [3]. Although there is no report yet available of vancomycin resistant E. faecium (VREfm) outbreak in Iran but there is ample evidence showing that the frequency of these strains are increasing in hospitals and therefore caused a major therapeutic concern in recent years $[4,5]$.

Characterization of VREfm strains is crucial for the effective management of infection caused by this organism

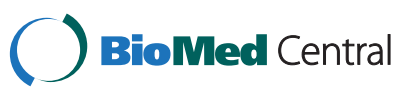


[6]. This characterization could be achieved with simple assays such as screening for resistance against antibiotics or more complex methods like Pulsed-field gel electrophoresis (PFGE) which is a powerful genotyping method used to study enterococcal distribution [7]. Routes of transmission and genetic linkage of these hospital isolates were examined in many epidemiological and molecular studies. Clonal spread of VREfm strains has been associated with disease outbreak in hospitals [7]. However, different studies in Iran suggest the heterogeneity and polyclonal distribution of nosocomial infection in hospital environments [8-10].

Detection of antibiotic resistance patterns of VRE isolates against a vast spectrum of antibiotics and understanding how these strains transmitted based on their genetic features could be useful in eliminating the infection in hospital. Therefore, the main objective of this study to investigating high frequency prevalence and antibiotic resistance patterns of VRE $f m$ isolates. We also analyzed the clonality and the genetic linkage between the strains by comparing their resistance patterns and pulsotypes in four major teaching hospitals in capital city Tehran, Iran.

\section{Materials and methods}

\section{Identification of bacterial strains}

The sampling was carried out in four major teaching hospitals in Tehran from September 2010 to June 2011. The isolates were obtained from clinical samples of indoor hospitalized patients (urine, wound, blood, abscess, sputum, bile, body fluid, intravenous catheter and trachea). All Enterococci isolates were identified according to their genus and species levels by gram staining, catalase reaction, growth in $6.5 \% \mathrm{NaCl}$, motility assessment, use of arabinose, bile and esculin hydrolysis and also pigment production after their growth on enterococcus selective agar (BBL, USA) all based on Falkman and Collins criteria [11]. PCR based study was conducted by using specific primer ( $\left.d d l_{\text {E.faecium }}\right)$ for each E.faecium species strains [12]. Protocols conformed to the ethical guidelines of the 1975 Declaration of Helsinki and were approved by Research Ethics Committee of the Tarbiat Modares University.

\section{DNA extraction and polymerase chain reaction}

DNAs from different bacterial isolates were extracted by using appropriate DNA extraction Kit (Sinagene, Iran).

For PCR amplification assay, specific primers of $d d l_{\text {E.faecium }}$ and vancomycin resistance genes $(\operatorname{van} \mathrm{A}$ and $\operatorname{van} \mathrm{B})$ were used as describe previously [12]. E. faecium ATCC 51559 and E. faecalis ATCC 51229 as vanA and vanB standard encoding strains were used.

\section{Antimicrobial susceptibility test}

Antimicrobial susceptibility test for isolates of $E$. faecium was performed against vancomycin $(30 \mu \mathrm{g})$, teicoplanin
$(30 \mu \mathrm{g})$, gentamicin $(120 \mu \mathrm{g})$, ampicillin $(10 \mu \mathrm{g})$, erythromycin $(15 \mu \mathrm{g})$, ciprofloxacin $(5 \mu \mathrm{g})$, tetracycline $(30 \mu \mathrm{g})$, chloramphenicol $(30 \mu \mathrm{g})$, nitroforantoin $(300 \mu \mathrm{g})$, quinopristindalfopristin (synercid) $(15 \mu \mathrm{g})$ and linezolide $(30 \mu \mathrm{g})$ (Mast, UK), by the disc diffusion method. Vancomycin, ampicillin and gentamicin MICs (Minimum inhibitory concentration) were determined by the agar dilution method. The results were interpreted according to the Clinical and Laboratory Standards Institute guidelines (CLSI- 2011).

\section{Pulsed-field gel electrophoresis}

Genomic typing of isolates was performed by PFGE. Genomic DNA was prepared in low melting agarose plugs as described by Saifi et al. [8]. Restriction enzyme SmaI (Roche, Manheim, Germany) was used to digest the DNAs in small slices of the agarose plugs. The plugs were placed in 1\% agarose (Invitrogen, USA) that was in $0.5 \% \mathrm{TBE}$ and were electrophoresed with switch times ramped from $5 \mathrm{~s}$ to $35 \mathrm{~s}$ at $6 \mathrm{~V}$ with a run time of 23 hours at $14^{\circ} \mathrm{C}$ and an angel 120 in the Bio-Rad CHEF-DRIII system. Salmonella cholerasuis serotype Branderup H9812 was used as the molecular size marker. The gels were stained with ethidium bromide and photographed under ultraviolet light. The banding patterns were clustered by unweighted paired group (UPGMA) method by Gelcompar II software version 4.0. Interpretation was done by using the guidelines set out previously [13].

\section{Results}

\section{Prevalence of E. faecium species}

A total of 222 enterococcal isolates samples were collected from clinical hospitalized patients. Overall, $41.4 \%$ $(\mathrm{n}=92), 51.3 \%(\mathrm{n}=114)$ and $7.2 \%(\mathrm{n}=16)$ of the isolates were confirmed as E. faecium, E. faecalis and other species of enterococci respectively. Results of PCR for the $d d l$ gene confirmed the biochemically identification in E. faecium isolates. Most of the clinical isolates belonged to urine $70.6 \%(\mathrm{n}=65)$ followed by wound samples $9.7 \%$ $(\mathrm{n}=9)$ (Figure 1).

\section{Antimicrobial susceptibility testing}

Antibiotic resistance analysis showed a high rate of vancomycin resistance $48.9 \%(n=45)$ in the isolates under study. More (35.5\%) VREfm were isolated from hospitals by local code number 1 . ICUs $(n=17,38 \%)$ and kidney transplant wards $(n=9,20 \%)$ have the larger number of these strains. All (100\%) VRE isolates were also resistant to ampicillin, gentamicin, ciprofloxacin erythromycin and teicoplanin, this was followed by tetracycline $(n=36$, $80 \%)$, nitroforantoin $(\mathrm{n}=32,71 \%)$, choleramphenicol $(\mathrm{n}=$ $8,18 \%)$, quinopristin-dalfopristin (synercid) $(n=6,13.3 \%)$ and linezolide $(\mathrm{n}=1,2 \%)$. MIC values for vancomycin, ampicillin and gentamicin were from 64 to 1024, 32 to 


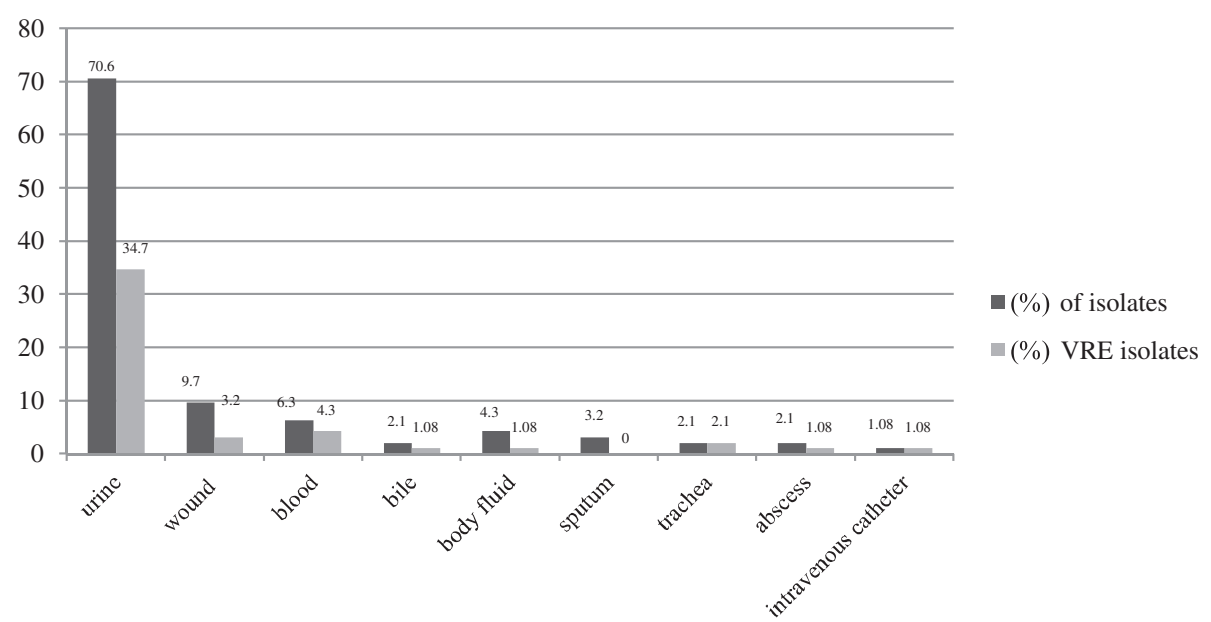

Figure 1 The rate (\%) of Enterococcus faecium strains isolated from clinical samples and (\%) of VRE.

256 and 512 to $1024 \mu \mathrm{g} / \mathrm{mL}$ respectively. $\mathrm{MIC}_{50}$ for vancomycin and ampicillin was $\geq 128 \mathrm{mg} / \mathrm{L}$ and for gentamicin was $\geq 1024 \mathrm{mg} / \mathrm{L}$. All VRE isolates harbored the vanA gene. Resistance to vancomycin, ampicillin, gentamicin, ciprofloxacin, tetracycline, erythromycin and teicoplanin was the dominant antibiotic resistance phenotype (77.7\%) $(\mathrm{n}=35)$. All VRE isolates were selected for genotyping by PFGE (Figure 2).

\section{Genotyping}

PFGE was performed in order to study the genetic linkage analysis among the clinical isolates. These isolates were divided into 17 pulsotype groups (A-Q) according to a similarity cutoff of $\geq 95 \%$. The predominant pulsotype (C) comprised 7 isolates (15\%). Six isolates (35.2\%) showed unique PFGE pattern as shown in Figure 2.

\section{Discussion}

Generally, E. faecalis has been known as the major cause of enterococcal infections, 10 times more prevalent than other enterococcus species. However, in recent years a remarkable change has seen from E. faecalis to E. faecium probably because of the emergence of VRE strains among members of this species in hospital environments [14]. The prevalence of E. faecium has been increased in Iranian hospitals during the last few years. In the present study, the ratio of infections due to E. faecalis to those caused by $E$. faecium was 1.2: 1 (51.3\% versus $41.4 \%)$ which is still higher than some previous reports published from Iran and some other countries $[4,15,16]$. The increased ratio was supported by enhancement of VREfm strains. The emergence of $E$. faecium isolates with a high level of resistance to three main classes of antibiotics (i.e. glycopeptides, $\beta$-lactams and amino glycosides) against enterococci spp is a major concern in hospitals. Congruent with the results from the USA hospitals, all the studied VRE isolates in Iran are resistant to ampicillin, whereas European hospital-derived clones are reported to be vancomycin susceptible but resistant to ampicillin and gentamicin [17-19].

Our current study clearly shows that in Iran the frequency distribution of VRE is high compared to rest of the countries of the world [20-23]. Also, all of VRE isolates showed resistance to more than 6 antibiotics, $60 \%$ of VRE isolates showed threatening resistance phenotype to vancomycin, teicoplanin, ampicillin, gentamicin, ciprofloxacin, tetracycline, erythromycin, nitrofourantoin (Va/Tei/Am/Gm/Cip/Te/E//Ni). Moreover high MIC values $\left(\mathrm{MIC}_{50} \geq 128\right)$ were also found for vancomycin and ampicillin as well as gentamicin $\mathrm{MIC}_{50} \geq 1024$.

It is also vibrant from the present study that all VRE isolates harbored vanA gene as describes previously. The wards related to kidney transplantation and nephrology, and ICU was estimated to be the ones with the highest risk of infection by VRE (Figure 2). The present study also reveals that linezolid and quinopristin- dalfopristin (synercid) were the most effective agents against the E. faecium isolates.

The dissemination of VRE $f m$, studies conducted in Iranian hospitals have found the dominancy in polyclonal among clinical isolates instead of clonal spreading as reported in USA and Europe but has consistency with Saudi Arabian hospitals [7,18-20].

The relative congruence of antibiotic resistance patterns and the specific PFGE patterns among the studied isolates demonstrates the presence of E. faecium strains with similar clone types in each of the hospitals e.g. pulsotypes $C$, $\mathrm{H}$ and $\mathrm{L}$ in hospital 1, pulsotype $\mathrm{A}$ in hospital 2, pulsotype $\mathrm{I}$ in hospital 3 and $\mathrm{J}$ in hospital 4 were identified.

The pulsotype patterns and resistance profiles suggest that there was an inter-hospital dissemination of pulsotype $\mathrm{F}$ and $\mathrm{D}$ (isolates were obtained from different sources 


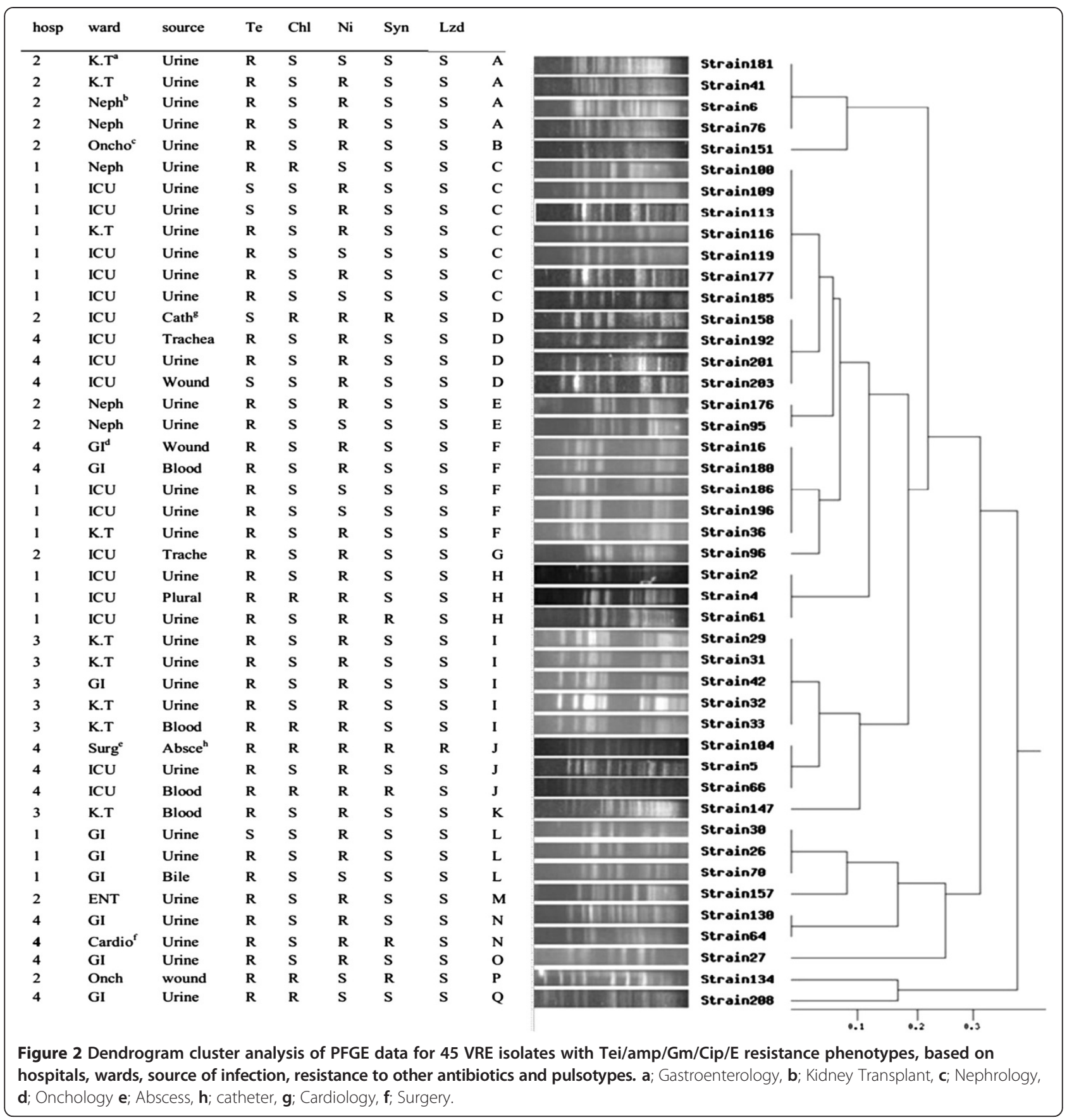

and wards). Most of the other pulsotypes (e.g. Pulsotypes A, C and I) were related to the same hospitals. The best example for intra hospital dissemination was pulsotype $C$, because all of these strains were isolated from urine samples and from the same ward of a hospital.

\section{Conclusions}

In conclusion, the increasing of polyclonal VRE $f m$ with threatening resistance phenotypes is a serious concern in Iranian hospitals. High resistance to antibiotics in our study was most probably due to the selective antibiotic pressures. The highlighted results need a strong attention for surveillance programs to continuously monitor the nosocomial changes in bacterial resistance and the exchange of information about pathogens.

Competing interests

The authors declare that they have no competing interests.

Authors' contributions

LS and AMM conceived the study. LS conducted the experiments and analyzed the results. LA and LS drafted the manuscript and made substantial 
contributions to the design of the study. LA, AMM, and TS critically reviewed the manuscript. MRZ, RR, TS and MA participated in data analysis. All the authors studied and approved the final manuscript.

\section{Acknowledgements}

This work was funded in part by a grant from Tarbiat Modares University and Research Center of Gastroenterology and Liver Diseases, Shahid Beheshti University of Medical Sciences, Tehran, Iran. We would like to thank all the staff at Food Born Disease Department of Taleghani hospital, ICUs in studied hospitals (Taleghani, Labbafinejad, Mofid and Loghman), and Molecular Biology Research Center, Baqiyatallah University of Medical Sciences of Tehran, Iran.

\section{Author details}

${ }^{1}$ Department of Bacteriology, Faculty of Medical Sciences, Tarbiat Modares University, P. O. Box: 14115-111, Tehran, Iran. ${ }^{2}$ Research Center of Gastroenterology and Liver Diseases Shahid Beheshti, University of Medical Sciences, Tehran, Iran. ${ }^{3}$ Molecular Biology Research Center, Baqiyatallah, University of Medical Sciences of Tehran, Tehran, Iran. ${ }^{4}$ Department of Internal Medicine II, University Hospital Freiburg, Freiburg, Germany. ${ }^{5}$ Faculty of Biology, Albert Ludwigs University of Freiburg, Freiburg, Germany.

Received: 17 July 2013 Accepted: 9 September 2013 Published: 2 October 2013

\section{References}

1. Sood S, Malhotra M, Das BK, Kapi A: Enterococcal infections \& antimicrobial resistance. Indian J Med Res 2008, 128:111-121.

2. Cohen M: Epidemiology of drug resistance: implications for a postantimicrobial era. Science 1992, 257:1050-1055.

3. Suppola JP, Kolho E, Salmenlinna S, Tarkka E, Vuopio-Varkila J, Vaara M: vanA and vanB incorporate into an endemic ampicillin-resistant vancomycinsensitive Enterococcus faecium strain: effect on interpretation of clonality. J Clin Microbiol 1999, 37:3934-3939.

4. Sharifi Y, Hassani A, Ghotaslou R, Varshochi M, Hasani A, Soroush MH, et al: Vancomycin-resistant enterococci among clinical isolates from Northwest Iran: identification of therapeutic surrogates. J Med Microbiol 2012, doi:10.1099/jmm.0.036780-0.

5. Feizabadi MM, Sayadi S, Shokrzadeh L, Parvin M, Yadegarnia D: Increase in prevalence of vancomycin resistant isolates of Enterococcous faecium at Labbafinejad hospital. Iran J Clin Infect Diseases 2008, 3(2):73-77.

6. Arias CA, Murray BE: Antibiotic-Resistant Bugs in the 21st Century - A Clinical Super-Challenge. N Engl J Med 2009, 360:439-443.

7. Willems R, Van Schaik W: Transition of Enterococcus faecium from commensal organism to nosocomial pathogen. J Future Microbiology 2009, 9:1125-1135.

8. Saifi M, Pourshafie MR, Sultan Dallal MM, Katouli M: Clonal group of high level gentamicin-resistant $E$. faecium isolated from municipial wastewater and clinical samples in Tehran, Iran. Lett Apple Microbio/ 2009, 49:160-165.

9. Pourshafie MR, Talebi M, Saifi M, Katouli M, Eshraghi S, Kuhn I, Mlolby R: Clonal heterogeneity of clinical isolates of vancomycin-resistant Enterococcus faecium with unique vanS. J Tropical Medicine and International Health 2008, 13(5):722-727.

10. Talebi M, Rahimi F, Katouli M, Mollby R, Pourshafie MR: Epidemiologic link between Wastewater and Human Vancomycin-Resistant Enterococcus faecium Isolates. Curr microbial 2008, 56:468-47.

11. Falkman RR, Collin MD: identification of Enterococcus species isolated from human infections by a conventional test scheme. J Clin Microbiol 1989, 27:731-734

12. Kariyama R, Mitsuhata R, Chow JW, Clewell DB, Kumon H: Simple and reliable multiplex PCR assay for surveillance, isolates of vancomycinresistant enterococci. J Clin Microbiol 2000, 38:3092-3095.

13. Tenover FC, Arbeit RD, Goering RV, Mickelsen PA, Murray BE, Persing DH, et al: Interpreting chromosomal DNA restriction patterns produced by pulsed field gel electrophoresis: criteria for bacterial strain typing. J Clin Microbiol 1995, 33:2233-2239.

14. Mundy LM, Sahm DF, Gilmore M: Relationships between enterococcal virulence and antimicrobial resistance. Clin Microbiol Rev 2000, 13:513-522.
15. Lester CH, Sandvang D, Olsen SS, Schonheyder HC, Jarlov JO, Bangsborg J: Emergence of ampicillin-resistant Enterococcus faecium in Danish hospitals. J Antimicrob Chemother 2008, 62:1203-1206.

16. Oteo J, Cuevas O, Navarro C, Aracil B, Campos J: Trends in antimicrobial resistance in 3469 enterococci isolated from blood (EARSS experience 2001-2006, Spain): increasing ampicillin resistance in Enterococcus faecium. J Antimicrob Chemother 2007, 59:1044-1045.

17. Cattoir V, Leclercq R: Twenty-five years of shared life with vancomycinresistant enterococci: is it time to divorce? J Antimicrob Chemother 2013, 68(4):731-742. Advance Access published December 2, 2012.

18. Nichol KA, Sill M, Laing NM, Johnson JL, Hoban DJ, Zhanel GG: Molecular epidemiology of urinary tract isolates of vancomycin-resistant Enterococcus faecium from North America. Int J Antimicrob Agents 2006, 27:392-396

19. Regt MJ, Wangen LE, Top J, et al: High acquisition and environmental contamination rates of CC17 ampicillin-resistant Enterococcus faecium in a Dutch hospital. J Antimicrob Chemother 2008, 62:1401-1406.

20. Khan M, Van der Wall M, Farrel DJ, Cossins L, Van Belkum A, Aldian A, Hays JP: Analysis of vanA vancomycin resistance Enterococcus faecium isolates from Saudi Arabian hospitals reveals the presence of clonal cluster 17 and two new Tn1546 lineage types. J Antimicrob chemother 2008, 62:279-283

doi:10.1186/1746-1596-8-163

Cite this article as: Shokoohizadeh et al: High frequency distribution of heterogeneous vancomycin resistant Enterococcous faecium (VREfm) in Iranian hospitals. Diagnostic Pathology 2013 8:163.

\section{Submit your next manuscript to BioMed Central and take full advantage of:}

- Convenient online submission

- Thorough peer review

- No space constraints or color figure charges

- Immediate publication on acceptance

- Inclusion in PubMed, CAS, Scopus and Google Scholar

- Research which is freely available for redistribution

Submit your manuscript at www.biomedcentral.com/submit
C) Biomed Central 